Algebraic 83 Geometric $\mathcal{T}$ opology

Volume 5 (2005) 537-562

Published: 19 June 2005

ATG

\title{
Yang-Baxter deformations of quandles and racks
}

\author{
Michael EisermanN
}

\begin{abstract}
Given a rack $Q$ and a ring $\mathbb{A}$, one can construct a Yang-Baxter operator $c_{Q}: V \otimes V \rightarrow V \otimes V$ on the free $\mathbb{A}$-module $V=\mathbb{A} Q$ by setting $c_{Q}(x \otimes y)=y \otimes x^{y}$ for all $x, y \in Q$. In answer to a question initiated by D.N. Yetter and P.J. Freyd, this article classifies formal deformations of $c_{Q}$ in the space of Yang-Baxter operators. For the trivial rack, where $x^{y}=x$ for all $x, y$, one has, of course, the classical setting of $\mathrm{r}$-matrices and quantum groups. In the general case we introduce and calculate the cohomology theory that classifies infinitesimal deformations of $c_{Q}$. In many cases this allows us to conclude that $c_{Q}$ is rigid. In the remaining cases, where infinitesimal deformations are possible, we show that higher-order obstructions are the same as in the quantum case.
\end{abstract}

AMS Classification 17B37; 18D10,20F36,20G42,57M25

Keywords Yang-Baxter operator, r-matrix, braid group representation, deformation theory, infinitesimal deformation, Yang-Baxter cohomology

\section{Introduction}

Following M. Gerstenhaber [17, an algebraic deformation theory should

- define the class of objects within which deformation takes place,

- identify infinitesimal deformations as elements of a suitable cohomology,

- identify the obstructions to integration of an infinitesimal deformation,

- give criteria for rigidity, and possibly determine the rigid objects.

In answer to a question initiated by P.J. Freyd and D.N. Yetter [16, we carry out this programme for racks (linearized over some ring $\mathbb{A}$ ) and their formal deformations in the space of $\mathbb{A}$-linear Yang-Baxter operators.

A rack is a set $Q$ with a binary operation, denoted $(x, y) \mapsto x^{y}$, such that $c_{Q}: x \otimes y \mapsto y \otimes x^{y}$ defines a Yang-Baxter operator on the free $\mathbb{A}$-module $\mathbb{A} Q$ (see Section 1 for definitions). For a trivial rack, where $x^{y}=x$ for all $x, y \in Q$, we see that $c_{Q}$ is simply the transposition operator. In this case the theory of 
quantum groups 17, 28, 23, 24, produces a plethora of interesting deformations, which have received much attention over the last 20 years. It thus seems natural to study deformations of $c_{Q}$ in the general case, where $Q$ is a non-trivial rack.

\section{Outline of results}

We first introduce and calculate the cohomology theory that classifies infinitesimal deformations of racks in the space of Yang-Baxter operators. In many cases this suffices to deduce rigidity. In the remaining cases, where infinitesimal deformations are possible, we show that higher-order obstructions do not depend on $Q$ : they are the same as in the classical case of quantum invariants. (See subsection 1.4 for a precise statement.)

Formal Yang-Baxter deformations of racks thus have an unexpectedly simple description: up to equivalence they are just r-matrices with a special symmetry imposed by the inner automorphism group of the rack. Although this is intuitively plausible, it requires a careful analysis to arrive at an accurate formulation. The precise notion of entropic r-matrices will be defined in subsection 1.3 .

With regards to topological applications, this result may come as a disappointment in the quest for new knot invariants. To our consolation, we obtain a complete and concise solution to the deformation problem for racks, which is quite satisfactory from an algebraic point of view.

Throughout our calculations we consider the generic case where the order $|\operatorname{Inn}(Q)|$ of the inner automorphism group of the rack $Q$ is invertible in the ground ring $\mathbb{A}$. We should point out, however, that certain knot invariants arise only in the modular case, where $|\operatorname{Inn}(Q)|$ vanishes in $\mathbb{A}$; see the closing remarks in Section [6.

\section{How this paper is organized}

In order to state the results precisely, and to make this article self-contained, Section 1 first recalls the notions of Yang-Baxter operators (subsection 1.1) and racks (subsection 1.2). We can then introduce entropic maps (subsection 1.3) and state our results (subsection 1.4). We also discuss some examples (subsection 1.5) and put our results into perspective by briefly reviewing related work (subsection 1.6). 
The proofs are given in the next four sections: Section2 introduces Yang-Baxter cohomology and explains how it classifies infinitesimal deformations. Section 3 calculates this cohomology for racks. Section 4 generalizes our classification from infinitesimal to complete deformations. Section 5 examines higher-order obstructions and shows that they are the same as in the classical case of quantum invariants. Section [6, finally, discusses some open questions.

\section{Review of basic notions and statement of results}

\section{$1.1 \quad$ Yang-Baxter operators}

Let $\mathbb{A}$ be a commutative ring with unit. In the sequel all modules will be $\mathbb{A}$ modules, and all tensor products will be formed over $\mathbb{A}$. For every $\mathbb{A}$-module $V$ we denote by $V^{\otimes n}$ the $n$-fold tensor product of $V$ with itself. The identity map of $V$ is denoted by $\mathrm{I}: V \rightarrow V$, and $\mathbf{I}=\mathrm{I} \otimes \mathrm{I}$ stands for the identity map of $V \otimes V$.

Definition 1 A Yang-Baxter operator on $V$ is an automorphism $c: V \otimes V \rightarrow$ $V \otimes V$ that satisfies the Yang-Baxter equation, also called braid relation,

$$
(c \otimes \mathrm{I})(\mathrm{I} \otimes c)(c \otimes \mathrm{I})=(\mathrm{I} \otimes c)(c \otimes \mathrm{I})(\mathrm{I} \otimes c) \quad \text { in } \quad \operatorname{Aut}_{\mathbb{A}}\left(V^{\otimes 3}\right) .
$$

This equation first appeared in theoretical physics, in a paper by C.N. Yang [29] on the many-body problem in one dimension, in work of R.J. Baxter [2, 3] on exactly solvable models in statistical mechanics, and later in quantum field theory [13] in connection with the quantum inverse scattering method. It also has a very natural interpretation in terms of Artin's braid groups [1, 4] and their tensor representations:

Remark 2 Recall that the braid group on $n$ strands can be presented as

$$
\mathrm{B}_{n}=\left\langle\sigma_{1}, \ldots, \sigma_{n-1} \mid \begin{array}{cc}
\sigma_{i} \sigma_{j}=\sigma_{j} \sigma_{i} & \text { for }|i-j| \geq 2 \\
\sigma_{i} \sigma_{j} \sigma_{i}=\sigma_{j} \sigma_{i} \sigma_{j} & \text { for }|i-j|=1
\end{array}\right\rangle,
$$

where the braid $\sigma_{i}$ performs a positive half-twist of the strands $i$ and $i+1$. In graphical notation, braids can conveniently be represented as in Figure 1.

Given a Yang-Baxter operator $c$, we can define automorphisms $c_{i}: V^{\otimes n} \rightarrow V^{\otimes n}$ for $i=1, \ldots, n-1$ by setting $c_{i}=\mathrm{I}^{\otimes(i-1)} \otimes c \otimes \mathrm{I}^{\otimes(n-i-1)}$. The Artin presentation ensures that there exists a unique braid group representation $\rho_{c}^{n}: \mathrm{B}_{n} \rightarrow \operatorname{Aut}_{\mathbb{A}}\left(V^{\otimes n}\right)$ defined by $\rho_{c}^{n}\left(\sigma_{i}\right)=c_{i}$. 
Here we adopt the following convention: braid groups will act on the left, so that composition of braids corresponds to composition of maps. The braid in Figure 1 for example, reads $\beta=\sigma_{1}^{-2} \sigma_{2}^{2} \sigma_{1}^{-1} \sigma_{2}^{1} \sigma_{1}^{-1} \sigma_{2}^{1}$; it is represented by the operator $\rho_{c}^{3}(\beta)=c_{1}^{-2} c_{2}^{2} c_{1}^{-1} c_{2}^{1} c_{1}^{-1} c_{2}^{1}$ acting on $V^{\otimes 3}$.
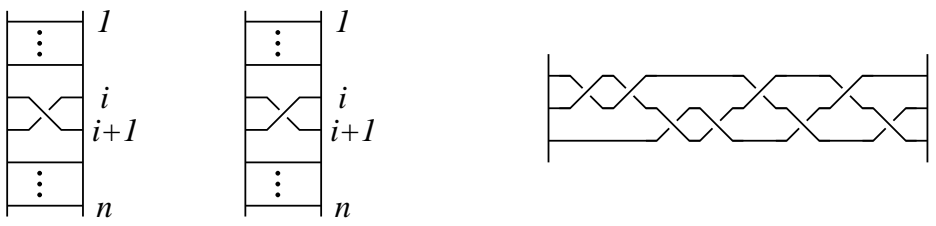

Figure 1: Elementary braids $\sigma_{i}^{+1}, \sigma_{i}^{-1}$; a more complex example $\beta$

Notice that Artin, after having introduced his braid groups, could have written down the Yang-Baxter equation in the 1920s, but without any non-trivial examples the theory would have remained void. It is a remarkable fact that the Yang-Baxter equation admits any interesting solutions at all. Many of them have only been discovered since the 1980s, and our first example recalls the most prominent one:

Example 3 For every $\mathbb{A}$-module $V$ the transposition $\tau: V \otimes V \rightarrow V \otimes V$ given by $\tau(a \otimes b)=b \otimes a$ is a Yang-Baxter operator. This in itself is not very surprising, but deformations of $\tau$ can be very interesting: Suppose that $V$ is free of rank 2 and choose a basis $(v, w)$. If we equip $V^{\otimes 2}$ with the basis $(v \otimes v, v \otimes w, w \otimes v, w \otimes w)$ then $\tau$ is represented by the matrix $c_{1}$ as follows:

$$
c_{1}=\left(\begin{array}{cccc}
1 & 0 & 0 & 0 \\
0 & 0 & 1 & 0 \\
0 & 1 & 0 & 0 \\
0 & 0 & 0 & 1
\end{array}\right), \quad c_{q}=\left(\begin{array}{cccc}
q & 0 & 0 & 0 \\
0 & 0 & q^{2} & 0 \\
0 & q^{2} & q-q^{3} & 0 \\
0 & 0 & 0 & q
\end{array}\right) .
$$

For every choice of $q \in \mathbb{A}^{\times}$, the matrix $c_{q}$ is a Yang-Baxter operator, and for $q=1$ we obtain the initial solution $c_{1}=\tau$. The family $\left(c_{q}\right)$, together with a suitable trace, yields the celebrated Jones polynomial [19, 20, 21], a formerly unexpected invariant of knots and links. More generally, deformations of $\tau$ lead to the so-called quantum invariants of knots and links.

Given the matrix $c_{q}$ of the preceding example, it is straightforward to check that it satisfies the Yang-Baxter equation. How to find such solutions, however, is a much harder question. Attempts to construct solutions in a systematic way have led to the theory of quantum groups (cf. [7]). For details we refer to the concise introduction [24] or the textbook [23]. 
Remark 4 A slight reformulation sometimes proves useful. Every YangBaxter operator $c$ can be written as $c=\tau f$ where $f: V \otimes V \rightarrow V \otimes V$ is an automorphism satisfying $f_{12} f_{13} f_{23}=f_{23} f_{13} f_{12}$, with $f_{i j}$ acting on the $i$ th and $j$ th factor of $V \otimes V \otimes V$. Such an operator $f$ is called an $r$-matrix. Depending on the context it may be more convenient to consider the r-matrix $f$ or the Yang-Baxter operator $\tau f$.

The set of Yang-Baxter operators is closed under conjugation by $\operatorname{Aut}(V)$, and conjugate operators yield conjugate braid group representations. A general goal of Yang-Baxter theory, as yet out of reach, would be to classify solutions of the Yang-Baxter equation modulo conjugation by $\operatorname{Aut}(V)$. In favourable cases this can be done at least locally, that is, one can classify deformations of a given Yang-Baxter operator. Our main result, as stated in subsection 1.4 below, covers a large class of such examples.

Definition 5 We fix an ideal $\mathfrak{m}$ in the ring $\mathbb{A}$. Suppose that $c: V \otimes V \rightarrow V \otimes V$ is a Yang-Baxter operator. A map $\tilde{c}: V \otimes V \rightarrow V \otimes V$ is called a Yang-Baxter deformation of $c$ (with respect to $\mathfrak{m}$ ) if $\tilde{c}$ is itself a Yang-Baxter operator and satisfies $\tilde{c} \equiv c$ modulo $\mathfrak{m}$.

The typical setting is the power series ring $\mathbb{A}=\mathbb{K} \llbracket h \rrbracket$ over a field $\mathbb{K}$, equipped with its maximal ideal $\mathfrak{m}=(h)$. In Example 3 we can choose $q \in 1+\mathfrak{m}$, which ensures that $c_{q}$ is a deformation of $\tau$ in the sense of the definition.

Definition 6 An equivalence transformation (with respect to the ideal $\mathfrak{m}$ ) is an automorphism $\alpha: V \rightarrow V$ with $\alpha \equiv \mathrm{I}$ modulo $\mathfrak{m}$. Two Yang-Baxter operators $c$ and $\tilde{c}$ are called equivalent (with respect to $\mathfrak{m}$ ) if there exists an equivalence transformation $\alpha: V \rightarrow V$ such that $\tilde{c}=(\alpha \otimes \alpha) c(\alpha \otimes \alpha)^{-1}$.

For every invertible element $s \in 1+\mathfrak{m}$ multiplication yields a deformation $s \cdot c$ of $c$. Such a rescaling, even though uninteresting, is in general not equivalent to $c$. A deformation $\tilde{c}$ of $c$ is called trivial if it is equivalent to $c$ or to a rescaling $s \cdot c$ by some constant factor $s \in 1+\mathfrak{m}$. We say that $c$ is rigid if every Yang-Baxter deformation of $c$ is trivial.

\subsection{Quandles and racks}

Racks are a way to construct set-theoretic solutions of the Yang-Baxter equation. To begin with, consider a group $G$ and a subset $Q \subset G$ that is closed under conjugation. This allows us to define a binary operation $*: Q \times Q \rightarrow Q$ by setting $x * y=y^{-1} x y$, which enjoys the following properties: 
(Q1) For every $x \in Q$ we have $x * x=x$.

(Q2) Every right translation $\varrho(y): x \mapsto x * y$ is a bijection. (right invertibility)

(Q3) For all $x, y, z \in Q$ we have $(x * y) * z=(x * z) *(y * z)$. (self-distributivity)

Such structures have gained independent interest since the 1980s when they have been applied in low-dimensional topology, most notably to study knots and braids. This is why a general definition has proven useful:

Definition 7 Let $Q$ be a set with a binary operation $*$. We call $(Q, *)$ a quandle if it satisfies axioms (Q1-Q3), and a rack if satisfies axioms (Q2-Q3).

The term "quandle" goes back to D. Joyce 22]. The same structure was called "distributive groupoid" by S.V. Matveev [27, and "crystal" by L.H. Kauffman 25]. Since quandles are close to groups, their applications in knot theory are in close relationship to the knot group. We should point out, however, that there exist many quandles that do not embed into any group.

Axioms (Q2) and (Q3) are equivalent to saying that every right translation $\varrho(y): x \mapsto x * y$ is an automorphism of $(Q, *)$. This is why such a structure was called automorphic set by E. Brieskorn [5]. The somewhat shorter term rack was preferred by R. Fenn and C.P. Rourke [14].

Definition 8 Let $Q$ be a rack. The subgroup of $\operatorname{Aut}(Q)$ generated by the family $\{\varrho(y) \mid y \in Q\}$ is called the group of inner automorphisms, denoted $\operatorname{Inn}(Q)$. Two elements $x, y \in Q$ are called behaviourally equivalent, denoted $x \equiv y$, if $\varrho(x)=\varrho(y)$.

We adopt the convention that automorphisms of a rack $Q$ act on the right, written $x^{\phi}$ or $x * \phi$, which means that their composition $\phi \psi$ is defined by $x^{(\phi \psi)}=\left(x^{\phi}\right)^{\psi}$ for all $x \in Q$. For $x, y \in Q$ we use the notation $x^{y}$ and $x * y$ indifferently.

P.J. Freyd and D.N. Yetter [16] considered the similar notion of crossed G-sets. Here the defining data is a set $Q$ equipped with a right action of a group $G$ and a map $\varrho: Q \rightarrow G$ such that $\varrho\left(x^{g}\right)=g^{-1} \varrho(x) g$. One easily verifies that this defines a rack $(Q, *)$ with $x * y=x^{\varrho(y)}$. Conversely, every rack $(Q, *)$ defines a crossed $G$-set by choosing the group $G=\operatorname{Inn}(Q)$ with its natural action on $Q$ and $\varrho: Q \rightarrow \operatorname{Inn}(Q)$ as above. Notice, however, that crossed $G$-sets are slightly more general than quandles, because the group $G$ acting on $Q$ need not be chosen to be $\operatorname{Inn}(Q)$.

Just as quandles generalize knot colourings, racks are tailor-made for braid colourings, see E. Brieskorn [5]. This brings us back to our main theme: 
Proposition 9 Given a rack $Q$, one can construct a Yang-Baxter operator $c_{Q}$ as follows: let $V=\mathbb{A} Q$ be the free $\mathbb{A}$-module with basis $Q$ and define

$$
c_{Q}: \mathbb{A} Q \otimes \mathbb{A} Q \rightarrow \mathbb{A} Q \otimes \mathbb{A} Q \quad \text { by } \quad x \otimes y \mapsto y \otimes(x * y) \quad \text { for all } \quad x, y \in Q .
$$

By construction, $c_{Q}$ is a Yang-Baxter operator: Axiom (Q2) ensures that $c_{Q}$ is an automorphism, while Axiom (Q3) implies the Yang-Baxter equation.

\section{$1.3 \quad$ Entropic maps}

In examining deformations of the operator $c_{Q}$ we will encounter certain maps $f: \mathbb{A} Q^{n} \rightarrow \mathbb{A} Q^{n}$ that respect the inner symmetry of the rack $Q$. To formulate this precisely, we introduce some notation.

Definition 10 Using graphical notation, a map $f: \mathbb{A} Q^{n} \rightarrow \mathbb{A} Q^{n}$ is called entropic with respect to $c_{Q}$ if it satisfies, for each $i=0, \ldots, n$, the following equation:

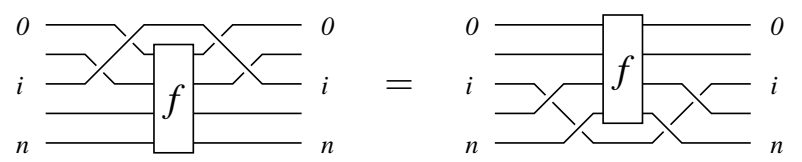

This can be reformulated in a more algebraic fashion. For notational convenience, we do not distinguish between the $\mathbb{A}$-linear map $f: \mathbb{A} Q^{n} \rightarrow \mathbb{A} Q^{n}$ and its matrix $f: Q^{n} \times Q^{n} \rightarrow \mathbb{A}$, related by the definition

$$
f:\left(x_{1} \otimes \cdots \otimes x_{n}\right) \mapsto \sum_{y_{1}, \ldots, y_{n}} f\left[\begin{array}{l}
x_{1}, \ldots, x_{n} \\
y_{1}, \ldots, y_{n}
\end{array}\right] \cdot\left(y_{1} \otimes \cdots \otimes y_{n}\right) .
$$

Matrix entries are thus denoted by $f\left[\begin{array}{l}x_{1}, \ldots, x_{n} \\ y_{1}, \ldots, y_{n}\end{array}\right]$ with indices $\left[\begin{array}{l}x_{1}, \ldots, x_{n} \\ y_{1}, \ldots, y_{n}\end{array}\right] \in Q^{n} \times Q^{n}$.

Definition 11 A map $f: \mathbb{A} Q^{n} \rightarrow \mathbb{A} Q^{n}$ is called quasi-diagonal if $f\left[\begin{array}{l}x_{1}, \ldots, x_{n} \\ y_{1}, \ldots, y_{n}\end{array}\right]=$ 0 whenever $x_{i} \not \equiv y_{i}$ for some index $i \in\{1, \ldots, n\}$. It is fully equivariant if it is equivariant under the action of $\operatorname{Inn}(Q)^{n}$, that is $f\left[\begin{array}{l}x_{1}, \ldots, x_{n} \\ y_{1}, \ldots, y_{n}\end{array}\right]=\left[\begin{array}{l}x_{1} * \alpha_{1}, \ldots, x_{n} * \alpha_{n} \\ y_{1} * \alpha_{1}, \ldots, y_{n} * \alpha_{n}\end{array}\right]$ for all $\alpha_{1}, \ldots, \alpha_{n} \in \operatorname{Inn}(Q)$.

Proposition 12 (proved in subsection 3.2) An $\mathbb{A}$-linear map $f: \mathbb{A} Q^{n} \rightarrow \mathbb{A} Q^{n}$ is entropic if and only if it is both quasi-diagonal and fully equivariant.

Remark 13 Entropic maps form a sub-algebra of $\operatorname{End}\left(\mathbb{A} Q^{n}\right)$. If $Q$ is trivial, then every map is entropic. If $\operatorname{Inn}(Q)$ acts transitively on $Q$ and $\varrho: Q \rightarrow \operatorname{Inn}(Q)$ is injective, then the only entropic maps are $\lambda$ id with $\lambda \in \mathbb{A}$. There are many examples in between the two extremes. Generally speaking, the larger $\operatorname{Inn}(Q)$ is, the fewer entropic maps there are. 


\subsection{Yang-Baxter deformations of racks}

As we have seen, each rack $Q$ provides a particular solution $c_{Q}$ to the YangBaxter equation. It appears natural to ask for deformations. Our main result solves this problem: under generic hypotheses, every Yang-Baxter deformation of $c_{Q}$ is equivalent to an entropic deformation.

Definition 14 Every deformation $c$ of $c_{Q}$ can be written as $c=c_{Q} f$ with $f \equiv \mathbb{I}$ modulo $\mathfrak{m}$. We call such a deformation entropic if $f$ is entropic.

The preliminaries being in place, we can now state the main results:

Theorem 15 (proved in Section 3) Consider the infinitesimal case where $\mathfrak{m}^{2}=0$. Then every entropic deformation of $c_{Q}$ satisfies the Yang-Baxter equation. If moreover $|\operatorname{Inn}(Q)|$ is invertible in $\mathbb{A}$, then every Yang-Baxter deformation of $c_{Q}$ is equivalent to exactly one entropic deformation.

Our approach to prove this theorem is classical: in the infinitesimal case everything becomes linear in first order terms, and the Yang-Baxter equation can be recast as a cochain complex. This can reasonably be called the Yang-Baxter cohomology. It is introduced in Section 2 and calculated in Section [3. Having this initial result at hand, we can proceed from infinitesimal to complete deformations:

Theorem 16 (proved in Section 4) Let $\mathbb{A}$ be a ring that is complete with respect to the ideal $\mathfrak{m}$, and let $Q$ be a rack such that $|\operatorname{Inn}(Q)|$ is invertible in $\mathbb{A}$. Then every Yang-Baxter deformation of $c_{Q}: \mathbb{A} Q^{2} \rightarrow \mathbb{A} Q^{2}$ is equivalent to an entropic deformation.

Notice that the hypotheses are always satisfied for a finite rack $Q$ over the complete local ring $\mathbb{A}=\mathbb{Q} \llbracket h \rrbracket$ with its maximal ideal $\mathfrak{m}=(h)$.

The preceding theorem ensures that we can restrict attention to entropic deformations; however, not every entropic deformation satisfies the Yang-Baxter equation. Being entropic suffices in the infinitesimal case, but in general higherorder terms introduce further obstructions. Quite surprisingly, they do not depend on $Q$ at all; higher-order obstructions are exactly the same as in the quantum case:

Theorem 17 (proved in Section 5) Consider a rack $Q$ and its associated Yang-Baxter operator $c_{Q}: \mathbb{A} Q^{2} \rightarrow \mathbb{A} Q^{2}$ over some ring $\mathbb{A}$. An entropic deformation $c_{Q} f$ satisfies the Yang-Baxter equation if and only if $\tau f$ satisfies the Yang-Baxter equation, that is, if and only if $f$ is an r-matrix. 
The transposition operator $\tau$ does not impose any infinitesimal restrictions; the only obstructions are those of degree 2 and higher. The preceding theorem says that entropic deformations of $c_{Q}$ are subject to exactly the same higher-order obstructions as deformations of $\tau$, plus the entropy condition enforced by a non-trivial inner automorphism group $\operatorname{Inn}(Q)$. In this sense, entropic YangBaxter deformations of $c_{Q}$ are just entropic r-matrices. We have thus reduced the theory of formal Yang-Baxter deformations of racks to the quantum case [7, 28, 23, 24].

\subsection{Applications and examples}

To simplify notation, we will consider here only quandles $Q$ that embed into some finite group $G$. This leads to certain classes of examples where deformations over $\mathbb{A}=\mathbb{Q} \llbracket h \rrbracket$ are particularly easy to understand.

Remark 18 Consider first a trivial quandle $Q$, with $x * y=x$ for all $x, y$, where $c_{Q}=\tau$ is simply the transposition operator. Here our results cannot add anything new: every map $f: \mathbb{A} Q^{n} \rightarrow \mathbb{A} Q^{n}$ is entropic, and so Theorem 15 simply restates that there are no infinitesimal obstructions (every deformation of $\tau$ satisfies the Yang-Baxter equation modulo $\mathfrak{m}^{2}$ ). There are, however, higherorder obstructions, which we have carefully excluded from our discussion: these form a subject of their own and belong to the much deeper theory of quantum invariants (see Example 3).

After the trivial quandle, which admits many deformations but escapes our techniques, let us consider the opposite case of a rigid operator:

Corollary 19 Let $G$ be a finite centreless group that is generated by a conjugacy class $Q$. Then every Yang-Baxter deformation of $c_{Q}$ over $\mathbb{Q} \llbracket h \rrbracket$ is equivalent to $s \cdot c_{Q}$ with some constant factor $s \in 1+(h)$. In other words, $c_{Q}$ is rigid.

Example 20 The smallest non-trivial example of a rigid operator is given by the set $Q=\{(12),(13),(23)\}$ of transpositions in the symmetric group $S_{3}$, or equivalently the set of reflections in the dihedral group $D_{3}$. Ordering the basis $Q \times Q$ lexicographically, we can represent $c_{Q}$ by the matrix

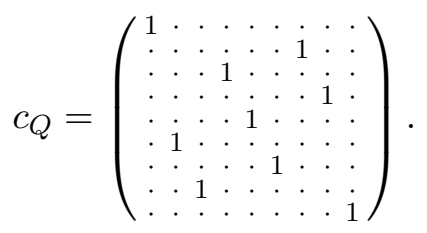

Algebraic 83 Geometric Topology, Volume 5 (2005) 
In the case of the Jones polynomial, the initial operator $\tau$ is trivial but the deformation $c_{q}$ is highly non-trivial. In the present example, the interesting part is the initial operator $c_{Q}$ itself: the associated link invariant is the number of 3-colourings, as defined by R.H. Fox. Unlike $\tau$, the Yang-Baxter operator $c_{Q}$ does not admit any non-trivial deformation over $\mathbb{Q} \llbracket h \rrbracket$. In this sense it is an isolated solution of the Yang-Baxter equation.

There are also racks in between the two extremes, which are neither trivial nor rigid. We indicate a class of examples where every infinitesimal deformation can be integrated, because higher-order obstructions miraculously vanish.

Corollary 21 Let $G$ be a finite group, generated by $Q=\cup_{i} Q_{i}$, where $Q_{1}, \ldots, Q_{n}$ are distinct conjugacy classes of $G$. Assume further that the centre $Z$ of $G$ satisfies $Z \cdot Q_{i}=Q_{i}$ for each $i=1, \ldots, n$. Then every Yang-Baxter deformation of $c_{Q}$ over $\mathbb{Q} \llbracket h \rrbracket$ is equivalent to one of the form $c(x \otimes y)=s_{i j} \cdot y \otimes x^{y}$ for $x \in Q_{i}$ and $y \in Q_{j}$, with constant factors $s_{i j} \in 1+h \mathbb{Q} \llbracket h \rrbracket[Z \times Z]$. Conversely, every deformation of this form satisfies the Yang-Baxter equation.

Example 22 Consider the set of reflections in the dihedral group $D_{4}$, that is

$$
Q=\{(13),(24),(12)(34),(14)(23)\}
$$

This set is closed under conjugation, hence a quandle. With respect to the lexicographical basis, $c_{Q}$ is represented by the following permutation matrix:

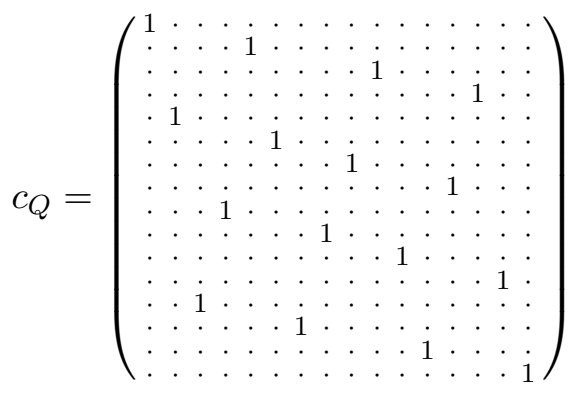

By construction, this matrix is a solution of the Yang-Baxter equation. According to Corollary 21] it admits a 16-fold deformation $c(\lambda)$ given by 


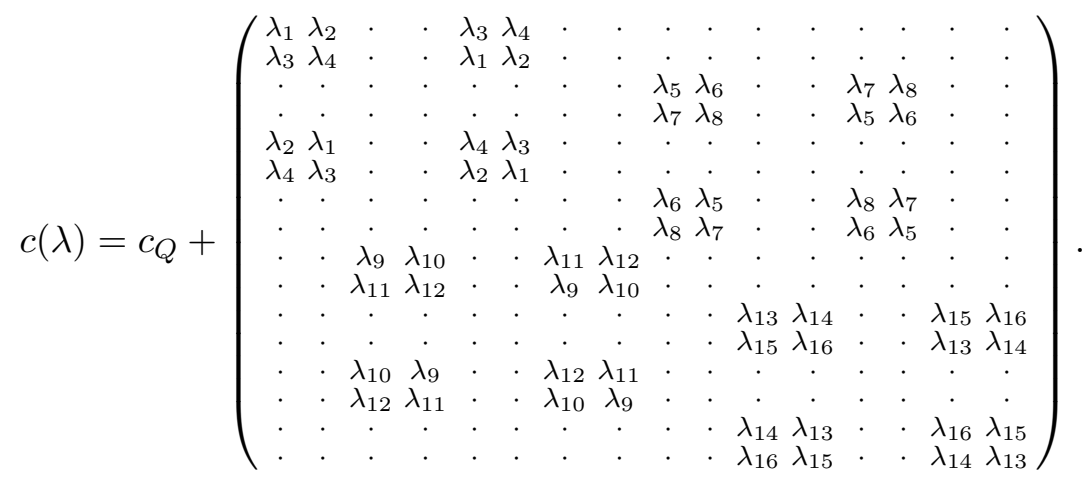

For every choice of parameters $\lambda_{1}, \ldots, \lambda_{16}$, the matrix $c(\lambda)$ satisfies the YangBaxter equation, and as a special case we get $c(0)=c_{Q}$. We finally remark that the trace of its square is given by

$$
\begin{aligned}
\operatorname{tr}\left[c(\lambda)^{2}\right] & =4\left(\lambda_{1}+1\right)^{2}+4 \lambda_{4}^{2}+4\left(\lambda_{13}+1\right)^{2}+4 \lambda_{16}^{2} \\
& +8\left(\lambda_{6}+1\right) \lambda_{11}+8\left(\lambda_{10}+1\right) \lambda_{7}+8 \lambda_{2} \lambda_{3}+8 \lambda_{14} \lambda_{15}+8 \lambda_{5} \lambda_{9}+8 \lambda_{8} \lambda_{12},
\end{aligned}
$$

which shows that none of the parameters can be eliminated by an equivalence transformation. This proves anew that the deformed operator $c(\lambda)$ is not equivalent to the initial operator $c_{Q}$.

Remark 23 It is amusing to note that the minimal Examples 3, 20, and 22 are the first three members of the family formed by reflections in dihedral groups. The following figure nicely summarizes the point:

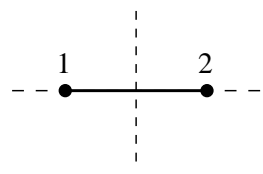

trivial but deformable

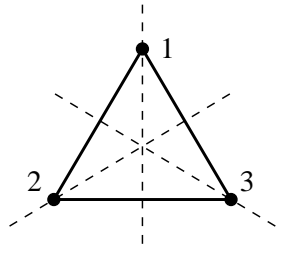

non-trivial but rigid

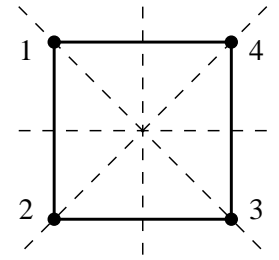

neither trivial nor rigid

Figure 2: The first three members of the dihedral family

\subsection{Related work}

Similar deformation and cohomology theories naturally arise in situations that are close or equivalent to the Yang-Baxter setting. 
- Our results can be reformulated in terms of deformations of modules over the quantum double $D(G)$ of a finite group $G$. In this form it has possibly been known to experts in quantum groups, but there seems to be no written account in the literature. See [24, ch. IX] for general background.

- The bialgebra approach was pursued by M. Gerstenhaber and S.D. Schack, who proved in [18, Section 8] that the group bialgebra $\mathbb{K} G$ is rigid as a bialgebra. They did not discuss deformations of its quantum double $D(G)$.

- Our approach can also be reformulated in terms of deformations of braided monoidal categories. This point of view was put forward by P.J. Freyd and D.N. Yetter in [16. The deformation of quandles and racks appeared as an example, but only diagonal deformations were taken into account.

- Diagonal deformations have been more fully developed in [6], where quandle cohomology was used to construct state-sum invariants of knots. P. Etingof and M. Graña [1] have calculated rack cohomology $H^{*}(Q, \mathbb{A})$ assuming $|\operatorname{Inn}(Q)|$ invertible in $\mathbb{A}$. Our calculation of $H_{\mathrm{YB}}^{2}\left(c_{Q}, \mathbb{A}\right)$ generalizes their result from diagonal to general Yang-Baxter deformations.

- In 30 Yetter considered deformations of braided monoidal categories in full generality; see also 31] and the bibliographical references therein. He was thus led to define a cohomology theory, which is essentially equivalent to Yang-Baxter cohomology. He did not, however, calculate any examples.

As far as I can tell, none of the previous results covers Yang-Baxter deformations of conjugacy classes, quandles, or racks.

\section{Yang-Baxter cohomology and infinitesimal defor- mations}

This section develops the infinitesimal deformation theory of Yang-Baxter operators. As usual, this is most conveniently formulated in terms of a suitable cohomology theory, which we will now define.

\subsection{Yang-Baxter cohomology}

Let $\mathbb{A}$ be a commutative ring with unit and let $\mathfrak{m}$ be an ideal in $\mathbb{A}$. Given an $\mathbb{A}$-module $V$ and a Yang-Baxter operator $c: V^{\otimes 2} \rightarrow V^{\otimes 2}$, we can construct a 
cochain complex of $\mathbb{A}$-modules $C^{n}=\operatorname{Hom}_{\mathbb{A}}\left(V^{\otimes n}, \mathfrak{m} V^{\otimes n}\right)$ as follows. Firstly, given $f \in C^{n}$, we define $d_{i}^{n} f \in C^{n+1}$ by

$$
d_{i}^{n} f=\left(c_{n} \cdots c_{i+1}\right)^{-1}(f \otimes \mathrm{I})\left(c_{n} \cdots c_{i+1}\right)-\left(c_{1} \cdots c_{i}\right)^{-1}(\mathrm{I} \otimes f)\left(c_{1} \cdots c_{i}\right)
$$

or in graphical notation:

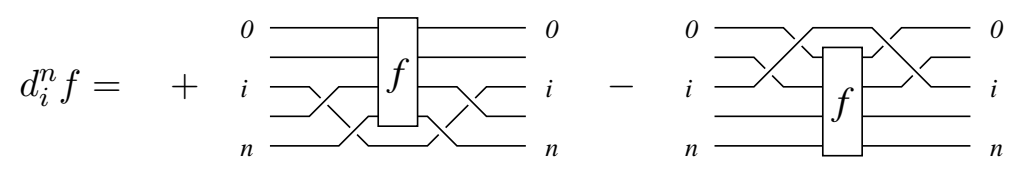

We then define the coboundary operator $d^{n}: C^{n} \rightarrow C^{n+1}$ by $d^{n}=\sum_{i=0}^{i=n}(-1)^{i} d_{i}^{n}$.

Proposition 24 The sequence $C^{1} \stackrel{d^{1}}{\longrightarrow} C^{2} \stackrel{d^{2}}{\longrightarrow} C^{3} \ldots$ is a cochain complex.

Proof The hypothesis that $c$ be a Yang-Baxter operator implies $d_{i}^{n+1} d_{j}^{n}=$ $d_{j+1}^{n+1} d_{i}^{n}$ for $i \leq j$. This can be proven by a straightforward computation; it is most easily verified using the graphical calculus suggested in the above figure. It follows, as usual, that terms cancel each other in pairs to yield $d^{n+1} d^{n}=0$.

Definition 25 We call $\left(C^{n}, d^{n}\right)$ the Yang-Baxter cochain complex associated with the operator $c$. As usual, elements of the kernel $Z^{n}=\operatorname{ker}\left(d^{n}\right)$ are called cocycles, and elements of the image $B^{n}=\operatorname{im}\left(d^{n-1}\right)$ are called coboundaries. The quotient $H^{n}=Z^{n} / B^{n}$ is called the Yang-Baxter cohomology of the operator $c$, denoted $H_{\mathrm{YB}}^{n}(c)$, or $H_{\mathrm{YB}}^{n}(c ; \mathbb{A}, \mathfrak{m})$ to indicate the dependence on the ring $\mathbb{A}$ and the ideal $\mathfrak{m}$.

Remark 26 A more general cohomology can be defined by taking coefficients in an arbitrary $\mathbb{A}$-module $U$. The operators $c_{i}$ act not only on $V^{\otimes n}$ but also on $U \otimes V^{\otimes n}$, extended by the trivial action on $U$. Using this convention, we can define a cochain complex $C^{n}=\operatorname{Hom}_{\mathbb{A}}\left(V^{\otimes n}, U \otimes V^{\otimes n}\right)$ with coboundary given by the same formulae as above.

Moreover, given a submodule $U^{\prime} \subset U$, we can consider the image of the induced map $U^{\prime} \otimes V^{\otimes n} \rightarrow U \otimes V^{\otimes n}$. (The image will be isomorphic with $U^{\prime} \otimes V^{\otimes n}$ if $V$ is flat.) Using this submodule instead of $U \otimes V^{\otimes n}$, we obtain yet another cohomology, denoted $H_{\mathrm{YB}}^{n}\left(c ; U, U^{\prime}\right)$. This generalizes our initial definition of $H_{\mathrm{YB}}^{n}(c ; \mathbb{A}, \mathfrak{m})$. All cohomology calculations in this article generalize verbatim to the case $\left(U, U^{\prime}\right)$. For our applications, however, it will be sufficient to consider the special case $(\mathbb{A}, \mathfrak{m})$. 


\subsection{Infinitesimal Yang-Baxter deformations}

Consider a Yang-Baxter operator $c: V^{\otimes 2} \rightarrow V^{\otimes 2}$. Every deformation $\tilde{c}: V^{\otimes 2} \rightarrow$ $V^{\otimes 2}$ of $c$ can be written as $\tilde{c}=c(\mathbb{I}+f)$ with perturbation term $f: V^{\otimes 2} \rightarrow$ $\mathfrak{m} V^{\otimes 2}$. For the rest of this section we will assume that $\mathfrak{m}^{2}=0$, which means that we consider infinitesimal deformations. One can always force this condition by passing to the quotient $\mathbb{A} / \mathfrak{m}^{2}$. The reason for this simplification is, of course, that higher-order terms are suppressed and everything becomes linear in first order terms.

Proposition 27 Suppose that the ideal $\mathfrak{m} \subset \mathbb{A}$ satisfies $\mathfrak{m}^{2}=0$. Then $\tilde{c}=$ $c(\mathbb{I}+f)$ is a Yang-Baxter operator if and only if $d^{2} f=0$. Moreover, $c$ and $\tilde{c}$ are equivalent via conjugation by $\alpha=\mathrm{I}+g$ with $g: V \rightarrow \mathfrak{m} V$ if and only if $f=d^{1} g$.

Proof Spelling out the Yang-Baxter equation for $\tilde{c}$ yields the Yang-Baxter equation for $c$ and six error terms of first order. More precisely, we obtain

$$
\begin{aligned}
& (\mathrm{I} \otimes \tilde{c})^{-1}(\tilde{c} \otimes \mathrm{I})^{-1}(\mathrm{I} \otimes \tilde{c})^{-1}(\tilde{c} \otimes \mathrm{I})(\mathrm{I} \otimes \tilde{c})(\tilde{c} \otimes \mathrm{I}) \\
& \quad=(\mathrm{I} \otimes c)^{-1}(c \otimes \mathrm{I})^{-1}(\mathrm{I} \otimes c)^{-1}(c \otimes \mathrm{I})(\mathrm{I} \otimes c)(c \otimes \mathrm{I})+d^{2} f .
\end{aligned}
$$

By hypothesis, $c$ is a Yang-Baxter operator, so the first term is the identity. As a consequence $\tilde{c}$ is a Yang-Baxter operator if and only if $f \in Z^{2}(c):=\operatorname{ker}\left(d^{2}\right)$.

On the other hand, given $\alpha=\mathrm{I}+g$ we have $\alpha^{-1}=\mathrm{I}-g$ and thus

$$
(\alpha \otimes \alpha)^{-1} c(\alpha \otimes \alpha)=c\left(\mathbb{I}+d^{1} g\right)
$$

As a consequence, $c$ and $\tilde{c}$ are equivalent if and only if $f \in B^{2}(c):=\operatorname{im}\left(d^{1}\right)$.

The infinitesimal deformations of $c$ are thus encoded in the cochain complex

$$
\operatorname{Hom}(V, \mathfrak{m} V) \stackrel{d^{1}}{\longrightarrow} \operatorname{Hom}\left(V^{\otimes 2}, \mathfrak{m} V^{\otimes 2}\right) \stackrel{d^{2}}{\longrightarrow} \operatorname{Hom}\left(V^{\otimes 3}, \mathfrak{m} V^{\otimes 3}\right) .
$$

Here $d^{1}$ maps each infinitesimal transformation $g: V \rightarrow \mathfrak{m} V$ to its infinitesimal perturbation term $d^{1} g: V^{\otimes 2} \rightarrow \mathfrak{m} V^{\otimes 2}$, which corresponds to an infinitesimally trivial deformation, and $d^{2}$ maps each infinitesimal perturbation $f: V^{\otimes 2} \rightarrow$ $\mathfrak{m} V^{\otimes 2}$ to its infinitesimal error term $d^{2} f: V^{\otimes 3} \rightarrow \mathfrak{m} V^{\otimes 3}$. By construction, we find again that $d^{2} \circ d^{1}=0$. We are interested in the quotient $\operatorname{ker}\left(d^{2}\right) / \operatorname{im}\left(d^{1}\right)$. 


\section{$3 \quad$ Yang-Baxter cohomology of racks}

This section will establish our main technical result: the explicit calculation of the second Yang-Baxter cohomology of a rack $(Q, *)$. As before, we consider the Yang-Baxter operator $c_{Q}: \mathbb{A} Q^{2} \rightarrow \mathbb{A} Q^{2}$ defined by $x \otimes y \mapsto y \otimes(x * y)$. We wish to study the associated cochain complex $C^{1} \rightarrow C^{2} \rightarrow C^{3} \rightarrow \ldots$ with cocycles $Z^{n}$ and coboundaries $B^{n}$. In degree 2 this is solved by the following theorem:

Theorem 28 Entropic $n$-cochains form a submodule of $Z^{n}$, denoted $E^{n}$. If the order of $\operatorname{Inn}(Q)$ is invertible in $\mathbb{A}$, then we have $Z^{2}=E^{2} \oplus B^{2}$, in other words, every 2-cocycle is cohomologous to exactly one entropic cocycle.

The theorem implies in particular that $H^{2} \cong E^{2}$, which is a perfectly explicit description of the second Yang-Baxter cohomology of a rack $Q$. The theorem does even a little better: in each cohomology class $\xi \in H^{2}$ it designates a preferred representative, namely the unique entropic cocycle in $\xi$. This will be proved by a sequence of four lemmas, which occupy the rest of this section.

\subsection{The coboundary operators}

Our goal is to calculate the Yang-Baxter cohomology of racks. Before doing so we will first make the coboundary operators more explicit by translating them from graphical to matrix notation.

Let $\delta: Q \times Q \rightarrow \mathbb{A}$ be the identity matrix, which in matrix notation is written as

$$
\delta\left[\begin{array}{l}
x \\
y
\end{array}\right]=\left\{\begin{array}{ll}
1 & \text { if } x=y \\
0 & \text { if } x \neq y
\end{array} .\right.
$$

In this notation the operator $d_{i}^{n} f: Q^{n+1} \times Q^{n+1} \rightarrow \mathfrak{m}$ is given by

$$
\begin{aligned}
\left(d_{i}^{n} f\right)\left[\begin{array}{l}
x_{0}, \ldots, x_{n} \\
y_{0}, \ldots, y_{n}
\end{array}\right]= & +f\left[\begin{array}{c}
x_{0}, \ldots, x_{i-1}, x_{i+1}, \ldots, x_{n} \\
y_{0}, \ldots, y_{i-1}, y_{i+1}, \ldots, y_{n}
\end{array}\right] \cdot \delta\left[\begin{array}{l}
x_{i}^{x_{i+1} \cdots x_{n}} \\
y_{i}^{y_{i+1} \cdots y_{n}}
\end{array}\right] \\
& -f\left[\begin{array}{c}
x_{0}^{x_{i}}, \ldots, x_{i-1}^{x_{i}}, x_{i+1}, \ldots, x_{n} \\
y_{0}^{y_{i}}, \ldots, y_{i-1}^{y_{i}}, y_{i+1}, \ldots, y_{n}
\end{array}\right] \cdot \delta\left[\begin{array}{l}
x_{i} \\
y_{i}
\end{array}\right] .
\end{aligned}
$$

The coboundary $d^{n} f: C^{n} \rightarrow C^{n+1}$ is given by $d^{n} f=\sum_{i=0}^{i=n}(-1)^{i} d_{i}^{n} f$. 
Remark 29 Our definitions were motivated by infinitesimal deformations in the space of Yang-Baxter operators. We could instead restrict all coboundary operators to diagonal matrices, that is, to matrices $f: Q^{n} \times Q^{n} \rightarrow \mathfrak{m}$ with $f\left[\begin{array}{l}x_{1}, \ldots, x_{n} \\ y_{1}, \ldots, y_{n}\end{array}\right]=0$ whenever $x_{i} \neq y_{i}$ for some $i$. In this case we obtain the cochain complex of quandle or rack cohomology (see [6, 9, 15]).

\subsection{Characterization of entropic maps}

Recall from Definition 10 that a map $f: \mathbb{A} Q^{n} \rightarrow \mathfrak{m} Q^{n}$ is entropic if and only if $d_{0} f=\cdots=d_{n} f=0$. The following lemma gives a useful reformulation:

Lemma 30 Given an $\mathbb{A}$-linear map $f: \mathbb{A} Q^{n} \rightarrow \mathfrak{m} Q^{n}$ and any $k \in\{0, \ldots, n\}$, we have $d_{k} f=\cdots=d_{n} f=0$ if and only if the following two conditions hold:

$$
\begin{aligned}
D_{k}: \quad f\left[\begin{array}{l}
x_{1}, \ldots, x_{n} \\
y_{1}, \ldots, y_{n}
\end{array}\right] & =0 \text { whenever } x_{i} \not \equiv y_{i} \text { for some } i>k, \text { and } \\
E_{k}: \quad f\left[\begin{array}{l}
x_{1}, \ldots, x_{n} \\
y_{1}, \ldots, y_{n}
\end{array}\right] & =f\left[\begin{array}{l}
x_{1}^{\alpha}, \ldots, x_{i}^{\alpha}, x_{i+1}, \ldots, x_{n} \\
y_{1}^{\alpha}, \ldots, y_{i}^{\alpha}, y_{i+1}, \ldots, y_{n}
\end{array}\right] \text { for all } \alpha \in \operatorname{Inn}(Q) \text { and } i \geq
\end{aligned}
$$

In particular, $f$ is entropic if and only if it is quasi-diagonal and fully equivariant.

Proof By equation (10), conditions $D_{k}$ and $E_{k}$ imply that $d_{k} f=\cdots=d_{n} f=$ 0 . To prove the converse, we proceed by a downward induction on $k=n, \ldots, 0$. Assume $d_{k} f=\cdots=d_{n} f=0$ and that $D_{k+1}$ and $E_{k+1}$ are true. We want to establish $D_{k}$ and $E_{k}$. First of all, we can suppose that $x_{k+2} \equiv y_{k+2}, \ldots$, $x_{n} \equiv y_{n}$; otherwise $D_{k}$ and $E_{k}$ are trivially satisfied because all terms vanish. In order to prove $D_{k}$, consider the case $x_{k+1} \not \equiv y_{k+1}$. Since $\varrho\left(x_{k+1}\right) \neq \varrho\left(y_{k+1}\right)$, there exists $w \in Q$ with such that $u=w * \varrho\left(x_{k+1}\right)^{-1}$ differs from $v=w *$ $\varrho\left(y_{k+1}\right)^{-1}$. We can thus choose $u \neq v$ with $u^{x_{k+1}}=v^{y_{k+1}}$ to obtain

$$
0=\left(d_{k} f\right)\left[\begin{array}{c}
x_{1}, \ldots, x_{k}, u, x_{k+1}, \ldots, x_{n} \\
y_{1}, \ldots, y_{k}, v, y_{k+1}, \ldots, y_{n}
\end{array}\right]=f\left[\begin{array}{c}
x_{1}, \ldots, x_{k}, x_{k+1}, \ldots, x_{n} \\
y_{1}, \ldots, y_{k}, y_{k+1}, \ldots, y_{n}
\end{array}\right] .
$$

In order to prove $E_{k}$, it suffices to consider $\alpha=\varrho(z)$ with $z \in Q$, since these automorphisms generate $\operatorname{Inn}(Q)$. Here we obtain

$$
\begin{aligned}
0 & =\left(d_{k} f\right)\left[\begin{array}{c}
x_{1}, \ldots, x_{k}, z, x_{k+1}, \ldots, x_{n} \\
y_{1}, \ldots, y_{k}, z, y_{k+1}, \ldots, y_{n}
\end{array}\right] \\
& =f\left[\begin{array}{c}
x_{1}, \ldots, x_{k}, x_{k+1}, \ldots, x_{n} \\
y_{1}, \ldots, y_{k}, y_{k+1}, \ldots, y_{n}
\end{array}\right]-f\left[\begin{array}{c}
x_{1}^{z}, \ldots, x_{k}^{z}, x_{k+1}, \ldots, x_{n} \\
y_{1}^{z}, \ldots, y_{k}^{z}, y_{k+1}, \ldots, y_{n}
\end{array}\right] .
\end{aligned}
$$

This establishes the induction step $k+1 \rightarrow k$ and completes the proof. 
Notice that in the preceding lemma we can choose the ideal $\mathfrak{m}=\mathbb{A}$; we thus obtain the characterization of entropic maps announced in Proposition 12.

\subsection{Entropic coboundaries vanish}

On our way to establish $Z^{2}=E^{2} \oplus B^{2}$, we are now in position to prove the easy part:

Lemma 31 If the order of the inner automorphism group $G=\operatorname{Inn}(Q)$ is not a zero-divisor in $\mathbb{A}$, then $E^{n} \cap B^{n}=\{0\}$.

Proof Consider a coboundary $f=d g$ that is entropic. We have to show that $f=0$. By the previous lemma, we know that $f$ is quasi-diagonal, hence we can assume that $x_{i} \equiv y_{i}$ for all $i$. The equation $f=d g$ then simplifies to

$$
\begin{aligned}
f\left[\begin{array}{l}
x_{1}, \ldots, x_{n} \\
y_{1}, \ldots, y_{n}
\end{array}\right]=\sum_{i=1}^{i=n}(-1)^{i-1} \delta\left[\begin{array}{c}
x_{i} \\
y_{i}
\end{array}\right] & \left(g\left[\begin{array}{c}
x_{1}, \ldots, x_{i-1}, x_{i+1}, \ldots, x_{n} \\
y_{1}, \ldots, y_{i-1}, y_{i+1}, \ldots, y_{n}
\end{array}\right]\right. \\
& \left.-g\left[\begin{array}{c}
x_{1} * x_{i}, \ldots, x_{i-1} * x_{i}, x_{i+1}, \ldots, x_{n} \\
y_{1} * y_{i}, \ldots, y_{i-1} * y_{i}, y_{i+1}, \ldots, y_{n}
\end{array}\right]\right)
\end{aligned}
$$

Using the equivariance under the action of $G^{n}$, we obtain

$$
\begin{aligned}
|G|^{n} \cdot f\left[\begin{array}{l}
x_{1}, \ldots, x_{n} \\
y_{1}, \ldots, y_{n}
\end{array}\right]=\sum_{\alpha \in G^{n}} f\left[\begin{array}{c}
x_{1}^{\alpha_{1}}, \ldots, x_{n}^{\alpha_{n}} \\
y_{1}^{\alpha_{1}}, \ldots, y_{n}^{\alpha_{n}}
\end{array}\right] \\
=\sum_{i=1}^{i=n}(-1)^{i-1} \delta\left[\begin{array}{c}
x_{i} \\
y_{i}
\end{array}\right] \sum_{\alpha \in G^{n}}\left(g\left[\begin{array}{c}
x_{1}^{\alpha_{1}}, \ldots, x_{i-1}^{\alpha_{i-1}}, x_{i+1}^{\alpha_{i+1}}, \ldots, x_{n}^{\alpha_{n}} \\
y_{1}^{\alpha_{1}}, \ldots, y_{i-1}^{\alpha_{i}}, y_{i+1}^{\alpha_{i+1}}, \ldots, y_{n}^{\alpha_{n}}
\end{array}\right]\right. \\
\left.\quad-g\left[\begin{array}{c}
x_{1}^{\alpha_{1}} * x_{i}^{\alpha_{i}}, \ldots, x_{i-1}^{\alpha_{i-1}} * x_{i}^{\alpha_{i}}, x_{i+1}^{\alpha_{i+1}}, \ldots, x_{n}^{\alpha_{n}} \\
y_{1}^{\alpha_{1}} * y_{i}^{\alpha_{i}}, \ldots, y_{i-1}^{\alpha_{i}} * y_{i}^{\alpha_{i}}, y_{i+1}^{\alpha_{i+1}}, \ldots, y_{n}^{\alpha_{n}}
\end{array}\right]\right)
\end{aligned}
$$

Fix some index $i$ in the outer sum. We can assume $x_{i}=y_{i}$, otherwise $\delta\left[\begin{array}{l}x_{i} \\ y_{i}\end{array}\right]=0$. Consider further some index $j<i$. The maps $x_{j} \mapsto x_{j}^{\alpha_{j}} * x_{i}^{\alpha_{i}}$ and $y_{j} \mapsto y_{j}^{\alpha_{j}} * y_{i}^{\alpha_{i}}$ correspond to the action of $\alpha_{j} \alpha_{i}^{-1} \varrho\left(x_{i}\right) \alpha_{i}$. As $\alpha_{j}$ runs through $G$, the product $\alpha_{j} \alpha_{i}^{-1} \varrho\left(x_{i}\right) \alpha_{i}$ also runs through $G$. This means that in the inner sum over $\alpha \in G^{n}$, all terms cancel each other in pairs. We conclude that $|G|^{n} f=0$, whence $f=0$. 


\subsection{Making cocycles equivariant by symmetrization}

Given an automorphism $\alpha \in \operatorname{Aut}(Q)$ and a cochain $f \in C^{n}$, we define the cochain $\alpha f \in C^{n}$ by

$$
(\alpha f)\left[\begin{array}{l}
x_{1}, \ldots, x_{n} \\
y_{1}, \ldots, y_{n}
\end{array}\right]:=f\left[\begin{array}{c}
x_{1}^{\alpha}, \ldots, x_{n}^{\alpha} \\
y_{1}^{\alpha}, \ldots, y_{n}^{\alpha}
\end{array}\right] .
$$

It is easily seen that $d(\alpha f)=\alpha(d f)$, hence $\alpha$ maps cocycles to cocycles, and coboundaries to coboundaries. The induced action on cohomology is denoted by $\alpha^{*}: H_{\mathrm{YB}}^{*}\left(c_{Q}\right) \rightarrow H_{\mathrm{YB}}^{*}\left(c_{Q}\right)$.

Lemma 32 Every inner automorphism $\alpha \in \operatorname{Inn}(Q)$ acts trivially on $H_{\mathrm{YB}}^{*}\left(c_{Q}\right)$. If the order of the inner automorphism group $G=\operatorname{Inn}(Q)$ is invertible in $\mathbb{A}$, then every cocycle is cohomologous to a $G$-equivariant cocycle.

Proof It suffices to consider inner automorphisms of the form $\alpha=\varrho(z)$ with $z \in Q$, since these automorphisms generate $\operatorname{Inn}(Q)$. For every cocycle $f \in Z^{n}$ we then have

$$
\begin{aligned}
f\left[\begin{array}{l}
x_{1}, \ldots, x_{n} \\
y_{1}, \ldots, y_{n}
\end{array}\right] & -f\left[\begin{array}{l}
x_{1}^{\alpha}, \ldots, x_{n}^{\alpha} \\
y_{1}^{\alpha}, \ldots, y_{n}^{\alpha}
\end{array}\right]=\left(d_{n}^{n} f\right)\left[\begin{array}{l}
x_{1}, \ldots, x_{n}, z \\
y_{1}, \ldots, y_{n}, z
\end{array}\right] \\
& =(-1)^{n} \sum_{i=0}^{n-1}(-1)^{i}\left(d_{i}^{n} f\right)\left[\begin{array}{l}
x_{1}, \ldots, x_{n}, z \\
y_{1}, \ldots, y_{n}, z
\end{array}\right]=\left(d^{n-1} g\right)\left[\begin{array}{l}
x_{1}, \ldots, x_{n} \\
y_{1}, \ldots, y_{n}
\end{array}\right]
\end{aligned}
$$

where the cochain $g \in C^{n-1}$ is defined by

$$
g\left[\begin{array}{l}
u_{1}, \ldots, u_{n-1} \\
v_{1}, \ldots, v_{n-1}
\end{array}\right]:=(-1)^{n} f\left[\begin{array}{l}
u_{1}, \ldots, u_{n-1}, z \\
v_{1}, \ldots, v_{n-1}, z
\end{array}\right] .
$$

This shows that $f-\alpha f=d g$, whence $\alpha$ acts trivially on $H_{\mathrm{YB}}^{*}\left(c_{Q}\right)$.

If the order of $G=\operatorname{Inn}(Q)$ is invertible in $\mathbb{A}$, then we can associate to each cochain $f$ a $G$-equivariant cochain $\bar{f}=\frac{1}{|G|} \sum_{\alpha \in G} \alpha f$. If $f$ is a cocycle then so is $\bar{f}$, and both are cohomologous by the preceding argument.

\subsection{Calculation of the second cohomology group}

Specializing to degree 2, the following lemma completes the proof of Theorem 28.

Lemma 33 Every equivariant 2-cocycle is cohomologous to an entropic one. 
Proof By hypothesis, we have $d^{2} f=0$, and according to Lemma 30 equivariance is equivalent to $d_{2}^{2} f=0$. We thus have $d_{0}^{2} f=d_{1}^{2} f$, or more explicitly:

$$
f\left[\begin{array}{l}
v, w \\
y, z
\end{array}\right]\left(\delta\left[\begin{array}{l}
u^{v w} \\
x^{y z}
\end{array}\right]-\delta\left[\begin{array}{l}
u \\
x
\end{array}\right]\right)=f\left[\begin{array}{l}
u, w \\
x, z
\end{array}\right] \delta\left[\begin{array}{l}
v^{w} \\
y^{z}
\end{array}\right]-f\left[\begin{array}{l}
u^{v}, w \\
x^{y}, z
\end{array}\right] \delta\left[\begin{array}{l}
v \\
y
\end{array}\right]
$$

for all $u, v, w, x, y, z \in Q$. It suffices to make $f$ quasi-diagonal, that is, to ensure $f\left[\begin{array}{l}v, w \\ y, z\end{array}\right]=0$ for $v \not \equiv y$ or $w \not \equiv z$. The left-hand side then vanishes identically, that is $d_{0}^{2} f=0$, which entails that the right-hand side also vanishes, whence $d_{1}^{2} f=0$.

First suppose that $w \not \equiv z$. Then there exists a pair $(v, y) \in Q \times Q$ with $v \neq y$ but $v^{w}=y^{z}$. If $(u, x) \in Q \times Q$ also satisfies $u \neq x$ and $u^{w}=x^{z}$, then Equation (2) implies that $f\left[\begin{array}{l}v, w \\ y, z\end{array}\right]=f\left[\begin{array}{l}u, w \\ x, z\end{array}\right]$. To see this, notice that $u^{w}=x^{z}$ is equivalent to $u^{v w}=x^{y z}$, because $\varrho(v) \varrho(w)=\varrho(w) \varrho\left(v^{w}\right)$ and $\varrho(y) \varrho(z)=\varrho(z) \varrho\left(y^{z}\right)$, with $v^{w}=y^{z}$ by our assumption. This allows us to define a 1 -cochain

$$
g\left[\begin{array}{l}
w \\
z
\end{array}\right]= \begin{cases}0 & \text { if } w \equiv z, \text { or else } \\
f\left[\begin{array}{l}
v, w \\
y, z
\end{array}\right] & \text { with } v \neq y \text { such that } v^{w}=y^{z} .\end{cases}
$$

According to the preceding argument, $g\left[\begin{array}{c}w \\ z\end{array}\right]$ is independent of the choice of $v, y$. In particular $g$ is equivariant since $f$ is. This implies $d_{1}^{1} g=0$, hence $d g=d_{0}^{1} g$ :

$$
(d g)\left[\begin{array}{l}
u, w \\
x, z
\end{array}\right]=g\left[\begin{array}{l}
w \\
z
\end{array}\right]\left(\delta\left[\begin{array}{l}
u^{w} \\
x^{z}
\end{array}\right]-\delta\left[\begin{array}{l}
u \\
x
\end{array}\right]\right)
$$

This vanishes whenever $w \equiv z$. Otherwise we choose $v \neq y$ with $v^{w}=y^{z}$ to obtain

$$
\begin{aligned}
(d g)\left[\begin{array}{l}
u, w \\
x, z
\end{array}\right] & =f\left[\begin{array}{l}
v, w \\
y, z
\end{array}\right]\left(\delta\left[\begin{array}{l}
u^{w} \\
x^{z}
\end{array}\right]-\delta\left[\begin{array}{l}
u \\
x
\end{array}\right]\right)=f\left[\begin{array}{l}
v, w \\
y, z
\end{array}\right]\left(\delta\left[\begin{array}{l}
u^{v w} \\
x^{y z}
\end{array}\right]-\delta\left[\begin{array}{l}
u \\
x
\end{array}\right]\right) \\
& =\left(d_{0}^{2} f\right)\left[\begin{array}{l}
u, v, w \\
x, y, z
\end{array}\right]=\left(d_{1}^{2} f\right)\left[\begin{array}{l}
u, v, w \\
x, y, z
\end{array}\right]=f\left[\begin{array}{l}
u, w \\
x, z
\end{array}\right] .
\end{aligned}
$$

By this construction, $\bar{f}:=f-d g$ is an equivariant cocycle satisfying $\bar{f}\left[\begin{array}{l}u, w \\ x, z\end{array}\right]=0$ whenever $w \not \equiv z$. For $\bar{f}$ our initial Equation (2) thus simplifies to

$$
\bar{f}\left[\begin{array}{l}
v, w \\
y, z
\end{array}\right]\left(\delta\left[\begin{array}{l}
u^{v} \\
x^{y}
\end{array}\right]-\delta\left[\begin{array}{l}
u \\
x
\end{array}\right]\right)=\left(\bar{f}\left[\begin{array}{l}
u, w \\
x, z
\end{array}\right]-\bar{f}\left[\begin{array}{l}
u^{v}, w \\
x^{y}, z
\end{array}\right]\right) \delta\left[\begin{array}{l}
v \\
y
\end{array}\right] .
$$

If $w \equiv z$ but $v \not \equiv y$, then choose $u \neq x$ with $u^{v}=x^{y}$ : the equation reduces to $\bar{f}\left[\begin{array}{l}v, w \\ y, z\end{array}\right]=0$. This shows that $\bar{f}$ is quasi-diagonal, in the sense that $\bar{f}\left[\begin{array}{c}v, w \\ y, z\end{array}\right]=0$ whenever $v \not \equiv y$ or $w \not \equiv z$. The left-hand side of our equation thus vanishes identically. The vanishing of the right-hand side is equivalent to $\bar{f}\left[\begin{array}{c}u, w \\ x, z\end{array}\right]=$ $\bar{f}\left[\begin{array}{l}u * \alpha, w \\ x * \alpha, z\end{array}\right]$ for all $\alpha \in \operatorname{Inn}(Q)$. This proves that $\bar{f}$ is an entropic cocycle, as desired. 
Proof of Theorem 28 The preceding lemmas allow us to conclude that $Z^{2}=$ $E^{2} \oplus B^{2}$, provided that the order of $G=\operatorname{Inn}(Q)$ is invertible in $\mathbb{A}$. Firstly, we have $E^{n} \cap B^{n}=\{0\}$ by Lemma 31. Moreover, every cocycle is cohomologous to a $G$-equivariant cocycle by Lemma 32 Finally, in degree 2 at least, every $G$ equivariant cocycle is cohomologous to an entropic cocycle, by Lemma 33 .

Question 34 Is it true that $Z^{n}=E^{n} \oplus B^{n}$ for all $n>2$ as well?

While all preceding arguments apply to $n$-cochains in arbitrary degree $n$, the present calculation of $H_{\mathrm{YB}}^{2}$ seems to work only for $n=2$. It is quite possible that some clever generalization will work for all $n$, but I could not figure out how to do this. This state of affairs, while not entirely satisfactory, seems acceptable because we use only the second cohomology in subsequent applications.

\section{Complete Yang-Baxter deformations}

In this section we will pass from infinitesimal to complete deformations. In order to do so, we will assume that the ring $\mathbb{A}$ is complete with respect to the ideal $\mathfrak{m}$, that is, we assume that the natural map $\mathbb{A} \rightarrow \lim \mathbb{A} / \mathfrak{m}^{n}$ is an isomorphism.

Theorem 35 Suppose that the ring $\mathbb{A}$ is complete with respect to the ideal $\mathfrak{m}$. Let $Q$ be a rack such that $|\operatorname{Inn}(Q)|$ is invertible in $\mathbb{A}$. Then every YangBaxter operator $c: \mathbb{A} Q^{2} \rightarrow \mathbb{A} Q^{2}$ with $c \equiv c_{Q}$ modulo $\mathfrak{m}$ is equivalent to an entropic deformation of $c_{Q}$. More explicitly, there exists $\alpha \equiv$ I modulo $\mathfrak{m}$ such that $(\alpha \otimes \alpha)^{-1} c(\alpha \otimes \alpha)=c_{Q} f$ with some entropic deformation term $f: \mathbb{A} Q^{2} \rightarrow \mathbb{A} Q^{2}, f \equiv \mathbb{I} \bmod \mathfrak{m}$.

The proof will use the usual induction argument for complete rings. We will first concentrate on the crucial inductive step: the passage from $\mathbb{A} / \mathfrak{m}^{n}$ to $\mathbb{A} / \mathfrak{m}^{n+1}$.

\subsection{The inductive step}

To simplify notation, we first assume that $\mathfrak{m}^{n+1}=0$. One can always force this condition by passing to the quotient $\mathbb{A} / \mathfrak{m}^{n+1}$.

Lemma 36 Consider a ring $\mathbb{A}$ with ideal $\mathfrak{m}$ such that $\mathfrak{m}^{n+1}=0$. Let $c: \mathbb{A} Q^{2} \rightarrow \mathbb{A} Q^{2}$ be a Yang-Baxter operator that satisfies $c \equiv c_{Q}$ modulo $\mathfrak{m}$ and is entropic modulo $\mathfrak{m}^{n}$. Then $c$ is equivalent to an entropic Yang-Baxter operator. More precisely, there exists $\alpha: \mathbb{A} Q \rightarrow \mathbb{A} Q$ with $\alpha \equiv$ I modulo $\mathfrak{m}^{n}$, such that $(\alpha \otimes \alpha)^{-1} c_{n}(\alpha \otimes \alpha)$ is an entropic deformation of $c_{Q}$. 
This lemma obviously includes and generalizes the infinitesimal case $n=1$, established in Theorem 28, upon which the following proof relies. The only new difficulty is that higher-order terms of the Yang-Baxter equation render the problem non-homogeneous. What is left, fortunately, is an affine structure:

Remark 37 Suppose $c: \mathbb{A} Q^{2} \rightarrow \mathbb{A} Q^{2}$ is a Yang-Baxter operator that satisfies $c \equiv c_{Q}$ modulo $\mathfrak{m}$ and is entropic modulo $\mathfrak{m}^{n}$. Let $X_{n}$ be the set of Yang-Baxter operators $\tilde{c}$ with $\tilde{c} \equiv c \bmod \mathfrak{m}^{n}$. For each $\tilde{c} \in X_{n}$ we have $\tilde{c}=c(\mathbb{I}+f)$, with $f: \mathbb{A} Q^{2} \rightarrow \mathfrak{m}^{n} Q^{2}$, and it is easily verified that $f \in Z^{2}\left(c_{Q} ; \mathbb{A}, \mathfrak{m}^{n}\right)$. Conversely, every $f \in Z^{2}\left(c_{Q} ; \mathbb{A}, \mathfrak{m}^{n}\right)$ yields a Yang-Baxter operator $c(\mathbb{I}+f) \in X_{n}$. In other words, $X_{n}$ is an affine space over $Z^{2}\left(c_{Q} ; \mathbb{A}, \mathfrak{m}^{n}\right)$.

Likewise, addition of a coboundary $f=d g \in B^{2}\left(c_{Q} ; \mathbb{A}, \mathfrak{m}^{n}\right)$ produces an equivalent deformation $\tilde{c}=c(\mathbb{I}+f)$. More explicitly, since $\mathfrak{m}^{n+1}=0$, the map $\alpha=\mathrm{I}+g$ has inverse $\alpha^{-1}=\mathrm{I}-g$; we thus obtain $\tilde{c}=(\alpha \otimes \alpha)^{-1} c(\alpha \otimes \alpha)$ as claimed.

Having the affine structure at hand, we can now proceed from $\mathbb{A} / \mathfrak{m}^{n}$ to $\mathbb{A} / \mathfrak{m}^{n+1}$ :

Proof of Lemma 36 As before let $X_{n}$ be the set of Yang-Baxter operators $\tilde{c}$ with $\tilde{c} \equiv c \bmod \mathfrak{m}^{n}$. Using the $Z^{2}$-affine structure on $X_{n}$, it suffices to find at least one entropic solution $\tilde{c} \in X_{n}$. Every other solution will then be of the form $\tilde{c}(\mathbb{I}+f)$ with $f \in Z^{2}\left(c_{Q} ; \mathbb{A}, \mathfrak{m}^{n}\right)$, hence equivalent to $\tilde{c}\left(\mathbb{I}+f^{\prime}\right)$ with some entropic $f^{\prime}$, according to Theorem 28. Since the composition of entropic maps is again entropic, this suffices to prove the lemma.

In order to find an entropic solution $\tilde{c} \in X_{n}$, we can first of all symmetrize $c$ : given $\alpha \in \operatorname{Inn}(Q)$, we have $\alpha c \in X_{n}$, because $c$ is equivariant modulo $\mathfrak{m}^{n}$. This implies that $\bar{c}=\frac{1}{|G|} \sum_{\alpha \in G} \alpha c$ lies in $X_{n}$, too. We thus obtain an equivariant operator $\bar{c}$, which we can write $\bar{c}=c_{Q}(\mathbb{I}+e)$ with deformation term $e: \mathbb{A} Q^{2} \rightarrow \mathfrak{m} Q^{2}$. In order to make $e$ quasi-diagonal, we decompose $e=f+g$ such that

$$
f\left[\begin{array}{l}
u, v \\
x, y
\end{array}\right]:= \begin{cases}e\left[\begin{array}{l}
u, v \\
x, y
\end{array}\right] & \text { if } u \equiv x, v \equiv y \\
0 & \text { otherwise }\end{cases}
$$

and

$$
g\left[\begin{array}{l}
u, v \\
x, y
\end{array}\right]:= \begin{cases}0 & \text { if } u \equiv x, v \equiv y \\
e\left[\begin{array}{l}
u, v \\
x, y
\end{array}\right] & \text { otherwise }\end{cases}
$$

By hypothesis, $e$ is quasi-diagonal modulo $\mathfrak{m}^{n}$, whence we have $e \equiv f \bmod \mathfrak{m}^{n}$ and $g: \mathbb{A} Q^{2} \rightarrow \mathfrak{m}^{n} Q^{2}$. We obtain by this construction a map $\tilde{c}=c_{Q}(\mathbb{I}+f)$ that is entropic and satisfies $\tilde{c} \equiv c \bmod \mathfrak{m}^{n}$. 
We claim that $\tilde{c}$ actually lies in $X_{n}$, that is, $\tilde{c}$ satisfies the Yang-Baxter equation. To see this, recall that $\bar{c}$ is a Yang-Baxter operator. For $\tilde{c}=\bar{c}(\mathbb{I}-g)$ we thus obtain

$$
(\mathrm{I} \otimes \tilde{c})^{-1}(\tilde{c} \otimes \mathrm{I})^{-1}(\mathrm{I} \otimes \tilde{c})^{-1}(\tilde{c} \otimes \mathrm{I})(\mathrm{I} \otimes \tilde{c})(\tilde{c} \otimes \mathrm{I})=-d^{2} g
$$

It is easy to check that the left-hand side is a quasi-diagonal map, whereas the right-hand side is zero on the quasi-diagonal. We conclude that both must vanish. This means that $\tilde{c}$ satisfies the Yang-Baxter equation, as claimed.

\subsection{From infinitesimal to complete deformations}

To conclude the passage from infinitesimal to complete, it only remains to put the ingredients together:

Proof of Theorem [35] Starting with $c_{1}:=c$ for $n=1$, suppose that $c_{n}=$ $c_{Q} f_{n}$ has a deformation term $f_{n}$ that is entropic modulo $\mathfrak{m}^{n}$. By Lemma 36. there exists $\alpha_{n}: \mathbb{A} Q \rightarrow \mathbb{A} Q$ with $\alpha_{n} \equiv \mathrm{I}$ modulo $\mathfrak{m}^{n}$, such that $c_{n+1}:=$ $\left(\alpha_{n} \otimes \alpha_{n}\right)^{-1} c_{n}\left(\alpha_{n} \otimes \alpha_{n}\right)$ is given by $c_{n+1}=c_{Q} f_{n+1}$ with $f_{n+1}$ entropic modulo $\mathfrak{m}^{n+1}$. (In fact, the lemma ensures that such a map $\alpha_{n}$ exists modulo $\mathfrak{m}^{n+1}$; this can be lifted to a map $\mathbb{A} Q \rightarrow \mathbb{A} Q$, which is again invertible because $\mathbb{A}$ is complete.) Completeness of $\mathbb{A}$ ensures that we can pass to the limit and define the infinite product $\alpha=\alpha_{1} \alpha_{2} \alpha_{3} \cdots$. By construction, $(\alpha \otimes \alpha)^{-1} c(\alpha \otimes \alpha)$ is entropic and equivalent to $c$, as desired.

\section{Entropic deformations and r-matrices}

As we have seen in the preceding theorem, every Yang-Baxter deformation of $c_{Q}$ over a complete ring $\mathbb{A}$ is equivalent to an entropic Yang-Baxter deformation. Conversely, however, not every entropic deformation gives rise to a Yang-Baxter operator: being entropic suffices in the infinitesimal case, but in general higherorder terms introduce further obstructions. Quite surprisingly, they do not depend on $Q$ at all:

Theorem 38 Consider a rack $Q$ and its Yang-Baxter operator $c_{Q}: \mathbb{A} Q^{2} \rightarrow$ $\mathbb{A} Q^{2}$ over some ring $\mathbb{A}$. An entropic deformation $\tilde{c}=c_{Q} f$ satisfies the YangBaxter equation if and only if $\tilde{\tau}=\tau f$ satisfies the Yang-Baxter equation, that is, if and only if $f$ is an r-matrix. 
As we have seen, the transposition operator $\tau$ does not impose any infinitesimal restrictions (the associated coboundary operator vanishes). The only obstructions are those of higher order. The preceding theorem says that the integration of an entropic infinitesimal deformation to a complete deformation of $c_{Q}$ entails exactly the same higher-order obstructions as in the quantum case 7, 28, 23, 24.

Proof The theorem follows once we have established the equation

$$
\begin{aligned}
(\mathrm{I} \otimes \tilde{c})^{-1}(\tilde{c} \otimes \mathrm{I})^{-1}(\mathrm{I} \otimes \tilde{c})^{-1}(\tilde{c} \otimes \mathrm{I})(\mathrm{I} \otimes \tilde{c})(\tilde{c} \otimes \mathrm{I}) & \\
& =(\mathrm{I} \otimes \tilde{\tau})^{-1}(\tilde{\tau} \otimes \mathrm{I})^{-1}(\mathrm{I} \otimes \tilde{\tau})^{-1}(\tilde{\tau} \otimes \mathrm{I})(\mathrm{I} \otimes \tilde{\tau})(\tilde{\tau} \otimes \mathrm{I}) .
\end{aligned}
$$

One way of proving this equality is by straightforward and tedious calculation. It seems more convenient, however, to employ a suitable graphical notation. Recall from Definition 10 that $f$ being entropic means

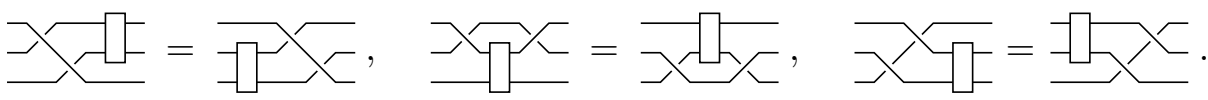

As before, positive and negative crossings represent $c_{Q}$ and $c_{Q}^{-1}$, respectively, whereas the box represents the deformation term $f$. The first and the last equation appear to be rather natural: they generalize the braid relation (or third Reidemeister move). The middle equation, however, is somewhat special and has a curious consequence:

For our operator $c_{Q}$ we know that the over-passing strand is not affected by a crossing. The middle equation thus implies that none of the strands is affected by the shown crossings: we could just as well replace them by transpositions!

Following this observation, our calculation boils down to verifying the following transformations:

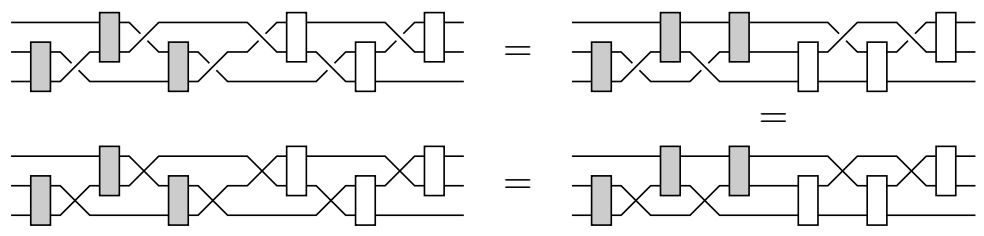

Here a white box represents the deformation $f$, whereas a shaded box represents its inverse $f^{-1}$. In the first line, positive and negative crossings represent $c_{Q}$ and $c_{Q}^{-1}$, respectively, whereas in the second line, crossings represent the transposition $\tau$. It is an easy matter to verify the equalities graphically, using the fact that $c_{Q}$ and $\tau$ are Yang-Baxter operators, and $f$ is entropic with respect to $c_{Q}$ and $\tau$. 


\section{Closing remarks and open questions}

Question 39 Our calculation in Section 3 relied on symmetrization, requiring that $|\operatorname{Inn}(Q)|$ be invertible in $\mathbb{A}$; this can be seen as the generic case of "coprime characteristic". It seems natural to investigate the cohomology $H_{\mathrm{YB}}^{*}\left(c_{Q} ; \mathbb{A}, \mathfrak{m}\right)$ in the "modular case", where $|\operatorname{Inn}(Q)|$ vanishes in the ring $\mathbb{A}$. Can one still find a succinct description of $H_{\mathrm{YB}}^{2}\left(c_{Q}\right)$, at least for certain (families of) examples? What are the higher order obstructions in this case? Do any interesting knot invariants arise in this way?

To illustrate the point, let us emphasize the connection with quandle and rack cohomology 6, 9, 15. Every map $\alpha: Q \times Q \rightarrow \mathbb{Z} / n \mathbb{Z}$ defines a diagonal deformation of $c_{Q}$ over $\mathbb{A}=\mathbb{Z}[h] /\left(n, h^{2}\right)$, with respect to the ideal $\mathfrak{m}=(h)$, by setting $c: x \otimes y \mapsto[1+h \alpha(x, y)] \cdot y \otimes x^{y}$. One easily checks that $c$ is a Yang-Baxter deformation of $c_{Q}$ if and only if $\alpha$ is an additive rack cocycle, that is

$$
\alpha(x, y)+\alpha\left(x^{y}, z\right)=\alpha(x, z)+\alpha\left(x^{z}, y^{z}\right) .
$$

Moreover, $c$ is equivalent to $c_{Q}$ if and only if $\alpha$ is a coboundary. Sample calculations [6] show that rack cohomology $H^{2}(Q, \mathbb{Z} / n \mathbb{Z})$ can be non-trivial. P. Etingof and M. Graña 11 have shown that this can only happen when $|\operatorname{Inn}(Q)|$ is noninvertible in $\mathbb{A}$. In these cases Yang-Baxter cohomology $H_{\mathrm{YB}}^{2}\left(c_{Q} ; \mathbb{A}, \mathfrak{m}\right)$ will include such extra deformations, and possibly non-diagonal ones, too.

Question 40 Is there a topological interpretation of the deformed invariants? Under suitable conditions, a Yang-Baxter deformation $c$ of a quandle $Q$ gives rise to invariants of knots and links 28, 23, 24. In the case of quandle cohomology one obtains so-called state-sum invariants [6], which have a natural interpretation in terms of knot group representations [10. Can a similar interpretation be established for Yang-Baxter deformations of $Q$ in general?

Question 41 What can be said about deformations of set-theoretic YangBaxter operators in general? Following [8, 12, 26], consider a set $Q$ equipped with a bijective map $c: Q \times Q \rightarrow Q \times Q$ satisfying $(c \times \mathrm{I})(\mathrm{I} \times c)(c \times \mathrm{I})=$ $(\mathrm{I} \times c)(c \times \mathrm{I})(\mathrm{I} \times c)$. Such a Yang-Baxter map gives rise to a right-action $Q \times Q \rightarrow Q,(x, y) \mapsto x^{y}$, as well as a left-action $Q \times Q \rightarrow Q,(x, y) \mapsto{ }^{x} y$, defined by $c(x, y)=\left({ }^{x} y, x^{y}\right)$. Notice that the case of a trivial left action, ${ }^{x} y=y$, corresponds precisely to racks.

As for racks, we can extend $c$ to an $\mathbb{A}$-linear map $c: \mathbb{A} Q^{2} \rightarrow \mathbb{A} Q^{2}$ and study deformations over $(\mathbb{A}, \mathfrak{m})$. As before, Yang-Baxter cohomology $H_{\mathrm{YB}}^{2}\left(c_{Q} ; \mathbb{A}, \mathfrak{m}\right)$ 
yields a convenient framework for the infinitesimal deformations of $c$, but con-

crete calculations are more involved. Does the cohomology $H_{\mathrm{YB}}^{2}(c)$ still correspond to entropic maps (under suitable hypotheses)? Can one establish similar rigidity properties? What happens in the modular case? Is there a topological interpretation?

\section{References}

[1] E Artin, Theory of braids, Ann. of Math. 48 (1947) 101-126 MathReview

[2] R J Baxter, Partition function of the eight-vertex lattice model, Ann. Physics 70 (1972) 193-228 MathReview

[3] R J Baxter, Exactly solved models in statistical mechanics, Academic Press Inc. London (1982) MathReview

[4] J S Birman, Braids, links, and mapping class groups, Ann. of Math. Studies 82, Princeton University Press, Princeton, N.J. (1974) MathReview

[5] E Brieskorn, Automorphic sets and braids and singularities, from: "Braids (Santa Cruz, CA, 1986)", Contemp. Math. 78, Amer. Math. Soc. Providence, RI (1988) 45-115 MathReview

[6] J S Carter, D Jelsovsky, S Kamada, L Langford, M Saito, Quandle cohomology and state-sum invariants of knotted curves and surfaces, Trans. Amer. Math. Soc. 355 (2003) 3947-3989 MathReview

[7] V G Drinfel'd, Quantum groups, from: "Proceedings of the International Congress of Mathematicians, Berkeley, Calif. 1986)", Amer. Math. Soc. Providence, RI (1987) 798-820 MathReview

[8] V G Drinfel'd, On some unsolved problems in quantum group theory, from: "Quantum groups (Leningrad, 1990)", Lecture Notes in Math. 1510, Springer, Berlin (1992) 1-8 MathReview

[9] M Eisermann, Homological characterization of the unknot, J. Pure Appl. Algebra 177 (2003) 131-157 MathReview

[10] M Eisermann, Knot colouring polynomials, preprint (2005)

[11] P Etingof, M Graña, On rack cohomology, J. Pure Appl. Algebra 177 (2003) 49-59 MathReview

[12] P Etingof, T Schedler, A Soloviev, Set-theoretical solutions to the quantum Yang-Baxter equation, Duke Math. J. 100 (1999) 169-209 MathReview

[13] L Faddeev, Integrable models in $(1+1)$-dimensional quantum field theory, from: "Recent advances in field theory and statistical mechanics (Les Houches, 1982)", North-Holland, Amsterdam (1984) 561-608 MathReview

[14] R Fenn, C Rourke, Racks and links in codimension two, J. Knot Theory Ramifications 1 (1992) 343-406 MathReview

[15] R Fenn, C Rourke, B Sanderson The rack space, Trans. Amer. Math. Soc. to appear, arXiv:math.GT/0304228 
[16] P J Freyd, D N Yetter, Braided compact closed categories with applications to low-dimensional topology, Adv. Math. 77 (1989) 156-182 MathReview

[17] M Gerstenhaber, On the deformation of rings and algebras, Ann. of Math. 79 (1964) 59-103 MathReview

[18] M Gerstenhaber, SD Schack, Algebras, bialgebras, quantum groups, and algebraic deformations, from: "Deformation theory and quantum groups with applications to mathematical physics (Amherst, MA, 1990)", Contemp. Math. 134, Amer. Math. Soc. Providence, RI (1992) 51-92 MathReview

[19] V F R Jones, A polynomial invariant for knots via von Neumann algebras, Bull. Amer. Math. Soc. (N.S.) 12 (1985) 103-111 MathReview

[20] V F R Jones, Hecke algebra representations of braid groups and link polynomials, Ann. of Math. 126 (1987) 335-388 MathReview

[21] V F R Jones, On knot invariants related to some statistical mechanical models, Pacific J. Math. 137 (1989) 311-334 MathReview

[22] D Joyce, A classifying invariant of knots, the knot quandle, J. Pure Appl. Algebra 23 (1982) 37-65 MathReview

[23] C Kassel, Quantum groups, Graduate Texts in Mathematics 155, SpringerVerlag, New York (1995) MathReview

[24] C Kassel, M Rosso, V Turaev, Quantum groups and knot invariants, volume 5 of Panoramas et Synthèses, Société Mathématique de France, Paris (1997) MathReview

[25] L H Kauffman, Knots and physics, Series on Knots and Everything 1, World Scientific Publishing Co. Inc. River Edge, NJ (2001) MathReview

[26] J Lu, M Yan, Y Zhu, On the set-theoretical Yang-Baxter equation, Duke Math. J. 104 (2000) 1-18 MathReview

[27] S V Matveev, Distributive groupoids in knot theory, Mat. Sb. (N.S.) 119(161) (1982) 78-88, 160; translation Math. USSR-Sb. 47 (1984) 73-83 MathReview

[28] V G Turaev, The Yang-Baxter equation and invariants of links, Invent. Math. 92 (1988) 527-553 MathReview

[29] C N Yang, Some exact results for the many-body problem in one dimension with repulsive delta-function interaction, Phys. Rev. Lett. 19 (1967) 1312-1315 MathReview

[30] D N Yetter, Topological quantum field theories associated to finite groups and crossed $G$-sets, J. Knot Theory Ramifications 1 (1992) 1-20 MathReview

[31] D N Yetter, Functorial knot theory, Series on Knots and Everything 26, World Scientific Publishing Co. Inc. River Edge, NJ (2001) MathReview

Institut Fourier, Université Grenoble I, 38402 St Martin d'Hères, France

Email: Michael.Eisermann@ujf-grenoble.fr

URL: WWW-fourier.ujf-grenoble.fr/ eiserm

Received: 16 September 2004 Revised: 18 May 2005 